\title{
On-orbit Performance of the ISS-CREAM Calorimeter
}

K.C. Kim ${ }^{1,2, *}$, Y. Amare ${ }^{1}$, D. Angelaszek ${ }^{1,2}$, N. Anthony ${ }^{1}$, G.H. Choi ${ }^{3}$, M. Chung ${ }^{1}$, M. Copley ${ }^{1}$, L. Derome ${ }^{4}$, L. Eraud ${ }^{4}$, C. Falana ${ }^{1}$, A. Gerrety ${ }^{1}$, L. Hagenau ${ }^{1}$, J.H. Han ${ }^{1}$, H.G. Huh ${ }^{1}$, Y.S. Hwang ${ }^{5}$, H.J. Hyun ${ }^{5}$, H.B. Jeon ${ }^{5}$, J.A. Jeon ${ }^{3}$, S. Jeong ${ }^{3}$, S.C. Kang ${ }^{5}$, H.J. Kim ${ }^{5}$, M.H. Kim ${ }^{1}$, H.Y. Lee ${ }^{3}$, J. Lee ${ }^{3,5}$, M.H. Lee', C. Lamb ${ }^{1}$, J. Liang ${ }^{1}$, L. Lu', J.P. Lundquist ${ }^{1,3}$, L. Lutz ${ }^{1}$, B. Mark ${ }^{1}$, A. Mechaca-Rocha ${ }^{6}$, T. Mernik $^{1}$, M. Nester $^{1}, 0$. Ofoha $^{1}$, H. Park ${ }^{5}$, I.H. Park ${ }^{3}$, J.M. Park ${ }^{5}$, N. Picot-Clemente ${ }^{1}$, S. Rostsky ${ }^{1}$, E.S. Seo $^{1,2}$, J.R. Smith ${ }^{1}$, R. Takeishi ${ }^{3}$, T. Tatoli ${ }^{1}$, P. Walpole ${ }^{1}$, R.P. Weinmann ${ }^{1}$, J. Wu ${ }^{1}$, Z. Yin $^{1,2}$, Y.S. Yoon ${ }^{1}$, H.G. Zhang ${ }^{1}$

${ }^{1}$ Inst. for Phys. Sci. and Tech., University of Maryland, College Park, MD, USA

${ }^{2}$ Dept. of Physics, University of Maryland, College Park, MD, USA

${ }^{3}$ Dept. of Physics, Sungkyunkwan University, Republic of Korea

${ }^{4}$ Laboratoire de Physique Subatomique et de Cosmologie, Grenoble, France

${ }^{5}$ Dept. of Physics, Kyungpook National University, Republic of Korea

${ }^{6}$ Instituto de Fisica, Universidad Nacional Autonoma de Mexico, Mexico

Cosmic Ray Energetics And Mass for the International Space Station (ISS-CREAM) experiment is designed to study the composition and energy spectra of cosmic-ray particles from $10^{12}$ to $10^{15}$ eV. ISS-CREAM was launched and deployed to the ISS in August 2017. The ISS-CREAM payload employs a Silicon Charge Detector for charge measurements, Top and Bottom Counting Detector for electron-hadron separation and a low-energy trigger, a Boronated Scintillator Detector for additional electron-hadron separation, and a Calorimeter (CAL) for energy measurements and a high-energy trigger. The CAL is constructed of 20 layers of tungsten plates interleaved with scintillating fiber ribbons read out by hybrid-photodiodes (HPDs) and densified carbon targets. Each CAL layer is made of $3.5 \mathrm{~mm}\left(1 \mathrm{X}_{0}\right)$ thick tungsten plates alternating with fifty $0.5 \mathrm{~mm}$ thick and $1 \mathrm{~cm}$ wide scintillating fiber ribbons. Consecutive layers of fiber ribbons are installed orthogonal to each other. Energy deposition in the CAL determines the particle energy and provides tracking information to determine which segment(s) of the charge detectors to use for the charge measurement. Tracking for showers is accomplished by extrapolating each shower axis back to the charge detectors. The performance of the ISS-CREAM CAL during flight is presented.

36th International Cosmic Ray Conference -ICRC2019-

July 24th - August 1st, 2019

Madison, WI, U.S.A.

${ }^{1}$ Speaker for the ISS-CREAM Collaboration ${ }^{\#}$

*E-mail: kckim@umd.edu

${ }^{\#}$ For the collaboration list see PoS(ICRC2019)1177 


\section{Introduction}

The ISS-CREAM instrument is a space-based experiment for the direct measurement of charged cosmic rays with energies up to $\sim 10^{15} \mathrm{eV}$. The science objectives of ISS-CREAM are to find answers to questions about the origin of "knee" in the all-particle cosmic-ray spectrum, the source of cosmic rays and the propagation history of cosmic rays in the interstellar medium. ISSCREAM will determine how the observed spectral differences between protons and heavier nuclei evolve at energies approaching the knee and measure detailed spectra of secondary nuclei that result from primary nuclei interacting with the interstellar medium. It will also search for spectral features such as a softening of the proton spectrum and accurately measure electrons to determine the possibility of nearby cosmic-ray sources. Further details on the ISS-CREAM project is presented elsewhere [1]. ISS-CREAM is an external payload which was installed on the International Space Station, specifically on Japanese Experimental Module Exposed Facility (JEM-EF). The one and half-year exposure allows an increase in the energy reach and reduction of uncertainties compared to previous balloon flights. The calorimeter is the primary detector that measures cosmic-ray particle energies. In this paper, the on-orbit performances of the calorimeter is presented.

\section{Instrument}

In order to achieve the science and measurement goals, several detectors with specific capabilities are required. The calorimeter (CAL) provides measurements of the incident energy of cosmic rays from $\sim 10^{12}$ to $\sim 10^{15} \mathrm{eV}$ with an energy resolution better than $50 \%[2,3]$. The silicon charge detector (SCD) provides measurements of particle charges to identify the elements from $1 \leq \mathrm{Z} \leq 26$ with a resolution of $0.2 \mathrm{e}$ to $0.3 \mathrm{e}[4,5]$. The top and bottom counting detectors (T/BCD) provide a low energy trigger and electron/hadron separation $[6,7]$. The boronated scintillator detector (BSD) detects the tail-end of the particle shower leaving the CAL and is sensitive to thermal neutrons generated within the CAL showers to provide additional separation between electrons and hadrons [8]. The overall payload configuration including the CAL, SCD, T/BCD, and BSD is shown in Figure 1.

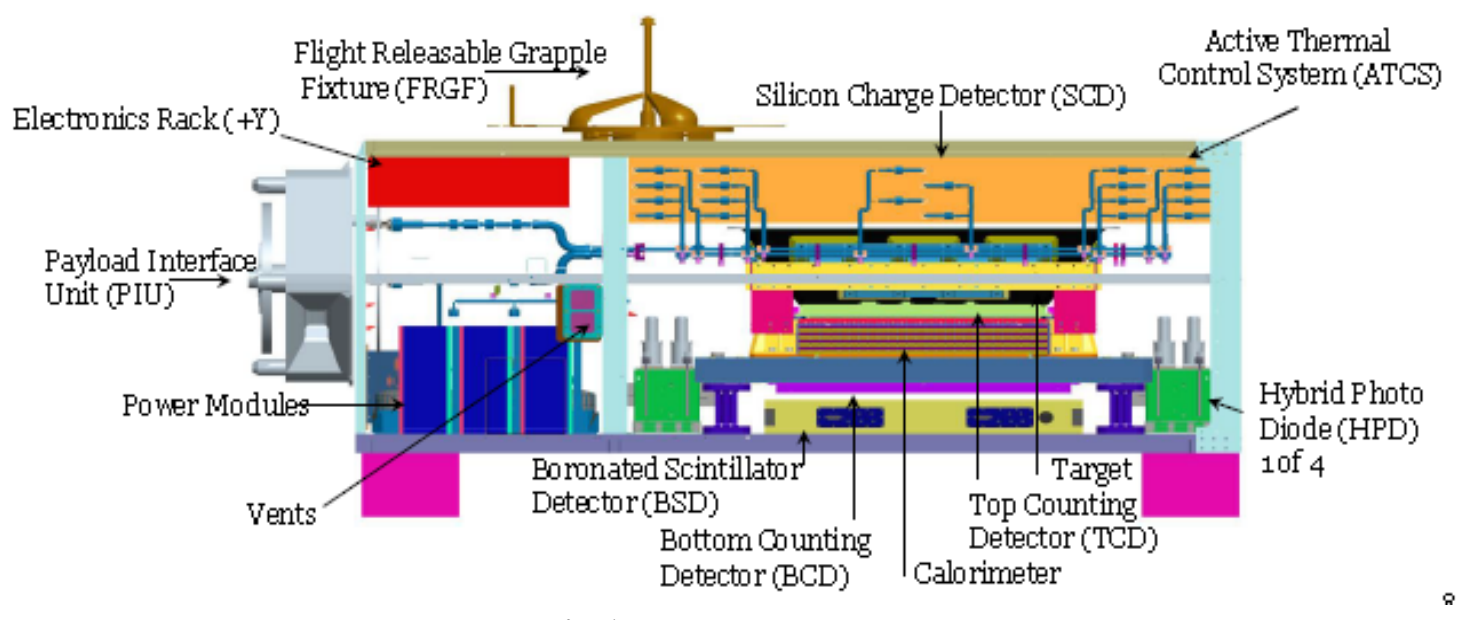

Fig.1. ISS-CREAM Instrument 


\section{Calorimeter (CAL)}

The ISS-CREAM CAL instrument provides a powerful method for measuring cosmic ray energies and is also used as an event trigger. The CAL, which is basically the same as CREAM $\mathrm{CAL}$, is a stack of 20 tungsten plates, each one radiation length $\left(\mathrm{X}_{0}\right)$ thick and with a surface area $50 \times 50 \mathrm{~cm}^{2}$, interleaved with layers of $1 \mathrm{~cm}$ wide and $50 \mathrm{~cm}$ long scintillating-fiber ribbons. Each ribbon is built by gluing together 19 scintillating fibers each $0.5 \mathrm{~mm}$ in diameter. The light signal from each ribbon is collected by means of an acrylic light mixer coupled to a bundle of 48 clear fibers where each fiber is distributed to low, mid, and high range channels. The threshold of each range for each channel is determined by electronic multi-charge gain runs. A total of 2560 channels are read out with forty Hybrid Photodiodes (HPDs). Ten HPDs are mounted on the top of a CAL readout box $[9,10]$.

For the ISS implementation, the CAL was modified to accommodate the increased mechanical forces due to transportation on the Space-X Falcon rocket as compared to balloon deployment. Some structural changes from the basic design of the CAL result from gluing together the tungsten layers and scintillating fiber ribbons, encasing the graphite target in a fiberglass shell, and redesigning the CAL and target mechanical support structure. The fiberglass sheets provide structural support for the epoxy to hold it within the area of the tungsten plates. Above and below the CAL layers are aluminum sheets that are glued to the tungsten-layer structure.

\subsection{On-orbit Performance of the CAL}

\subsubsection{Pedestal Noise and Charge Gain}

To set sparsification thresholds and pedestal subtraction, pedestal runs were performed once every hour for the CAL throughout the flight, leading to a pedestal value drift of less than 1 ADC count between two consecutive pedestal runs. The sparsification thresholds were automatically adjusted as needed following each pedestal run. Figure 2 shows the temperature and pedestal mean values of a CAL readout electronics as a function of time from 8/22/2017 to $5 / 2 / 2018$. Green dots represent the temperature and blue dots represent the pedestal mean values. The temperature stayed within the required operational range with daily variation of only a few ${ }^{\circ} \mathrm{C}$. The sinusoidal periodic behavior is due to the solar beta angle changes. The pedestal mean values are well correlated with the temperature. Figure 3 shows a distribution of pedestal RMS noise (in ADC units) for all $2560 \mathrm{CAL}$ readout channels. Except a small excess at the high end, it follows a Gaussian distribution, indicating a well controlled noise level. The mean value of 9.4 ADC units is fairly consistent with the pre-flight measurements on the ground. The pedestal RMS values of the CAL electronic channels were stable within 9.0 10.5 ADC during the flight.

The charge-injection run is used for gain calibration. A known amount of charge is injected into the front-end electronics to calibrate the response of the electronics channel. The gain of each electronics channel is defined as the ratio of the final ADC value of the channel to 
the input charge to the channel. The stability of the gain values is also monitored by having charge gain runs bi-hourly to identify any short- or long-term changes in the full electronics readout chain during the flight. Figure 4 shows an example data from a charge gain run. The mean charge gain of most channels stayed around 320 ADC/DAC during the flight. With multi-charge gain runs, we can decide threshold values for low-, mid-, and high-range signals for each ribbon.

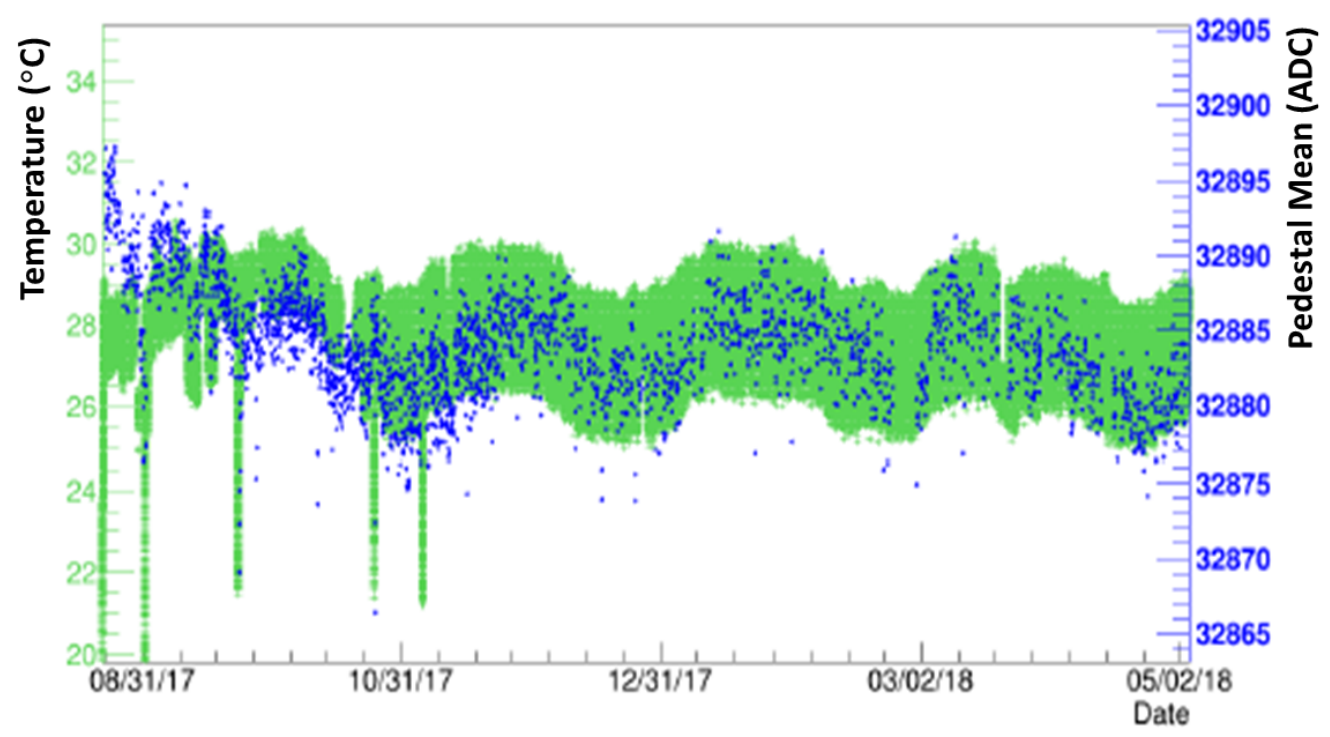

Fig.2. The temperature and pedestal mean values of a CAL readout electronics as a function of time. Green symbols represent the temperature of a mother board, and the dot symbols represent the pedestal mean values.

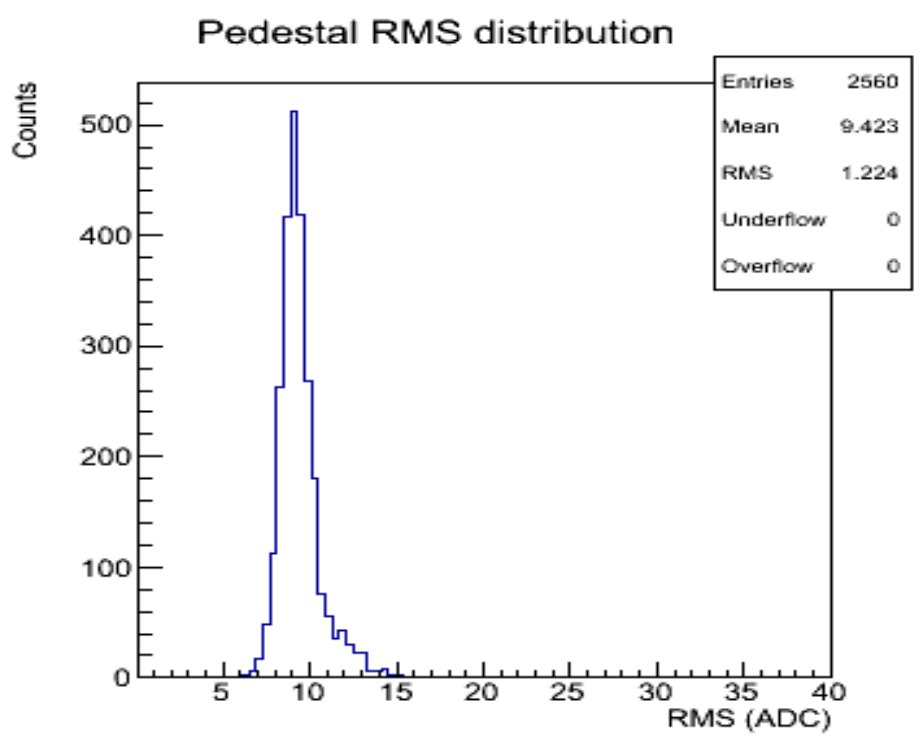

Fig. 3. Pedestal RMS distribution of all 2560 channels. 


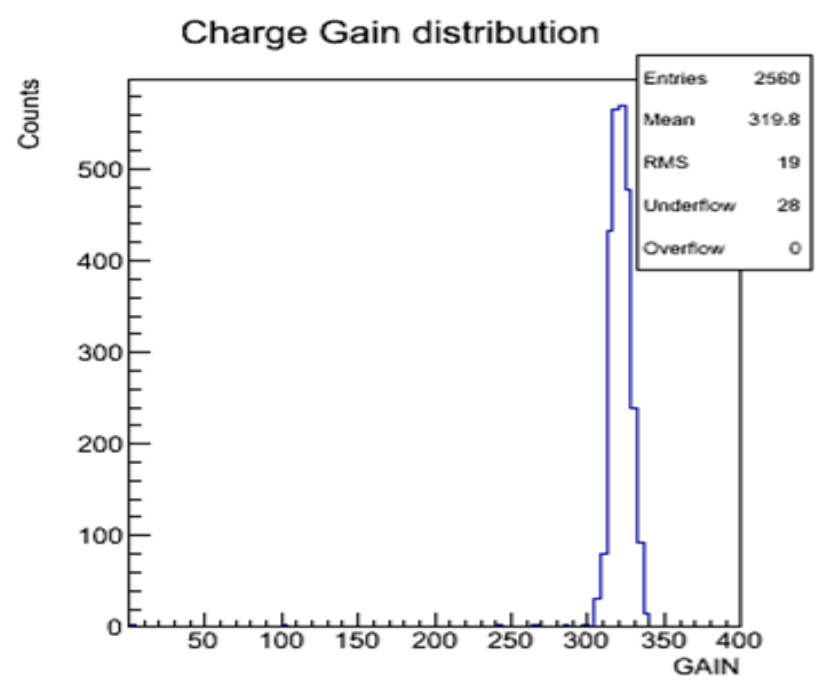

Fig.4. A CAL charge gain run of all channels.

\subsubsection{Event Display}

During the flight the instrument electronics mapping was confirmed by displaying science events as illustrated in Fig. 5. In this example of a Elow triggered event, hits in each detector, from the top, four layers of the SCD (i.e., SCD-1, $-2,-3$, and -4), TCD, CAL and BCD, clearly indicate a cosmic-ray particle track. The trajectory of the event was reconstructed from a linear fit to the core of the shower axis through the multiple layers of scintillating fiber strips in the CAL. The extrapolation of this reconstructed trajectory to the SCD matches well with the track in the SCD-1, -2, and -3, as well as TCD and BCD. There is no signal in SCD-4 because it was off when this example event was taken due to unstable power. The silicon pixel at the extrapolated position was selected for the charge measurement. Details of the data analysis can be found in Takeishi et al [11]. More advanced tracking algorithm is developed as reported by Lundquist et al. [12].

\subsubsection{Trigger}

The CAL trigger was designed to generate a trigger signal whenever a set of $\mathrm{N}$ consecutive CAL active layers ( $\mathrm{N}=4,6,8,10$, adjustable by command) each observed a signal exceeding a threshold that could be adjusted separately per half-layer by command. During the flight the CAL EHi trigger was defined to require six consecutive layers with at least one ribbon in each layer above a threshold value. The thresholds were scanned for a charge input and confirmed to be consistent with the pre-flight testing on the ground. The trigger logic was also tested using noise scanning thresholds for various algorithms. A sum of all the low range ADC values are compared for two different threshold setting in Fig. 6. The solid line represents -35 DAC and the dashed line represents higher threshold -75 DAC. TCD and BCD provide another trigger which is sensitive to lower energies than the CAL trigger using the number of hits in the TCD and BCD. For the Elow trigger one hit in TCD and two hits in BCD are required. 
(a)

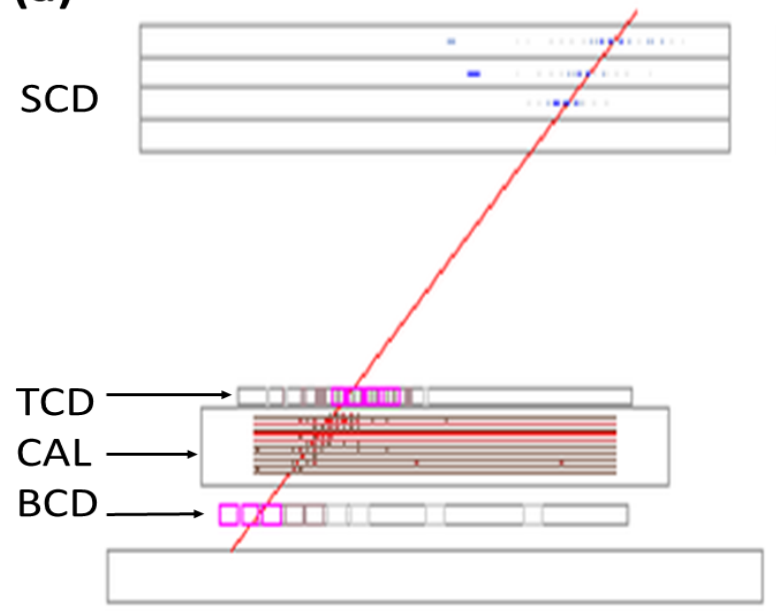

(b)

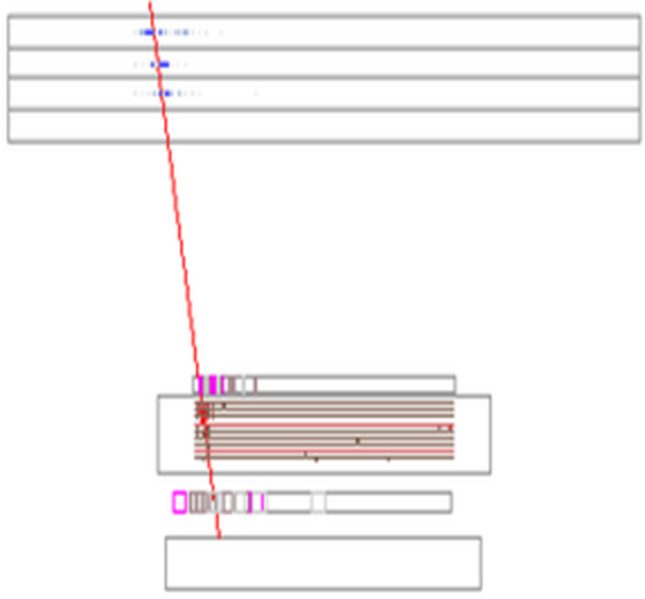

Fig. 5. A display of a cosmic ray event traverssing the ISS-CREAM instrument with (a) $X-Z$ and (b) Y-Z views. The blue squares are hits in the SCD. The pink squares are hits in the TCD and $\mathrm{BCD}$. The red squares are hits in the CAL. The red line crossing the instrument represents the trajectory of the event which was reconstructed from a linear fit to the core of the shower axis in the CAL.

\subsection{Data from the flight}

The CAL was calibrated with electrons ranging from 50 to $200 \mathrm{GeV}$ at CERN's H2 beam line in a series of beam tests. Each of 1,000 fiber ribbons were exposed to particle beams by scanning the CAL in the $\mathrm{x}$ and $\mathrm{y}$ directions. The energy conversion factors were obtained for all 1,000 ribbons using the same method as previous calibration runs [13-15]. Figure 7 shows the preliminary distribution of the total energy deposit in the CAL. The solid line histogram is for the EHi triggered events, and the dashed line histogram is for the Elow triggered events. The data show a reasonable power-law behavior as illustrated with a thicker solid line. The ADC to energy conversion is preliminary based on the calibration using only the low range values.

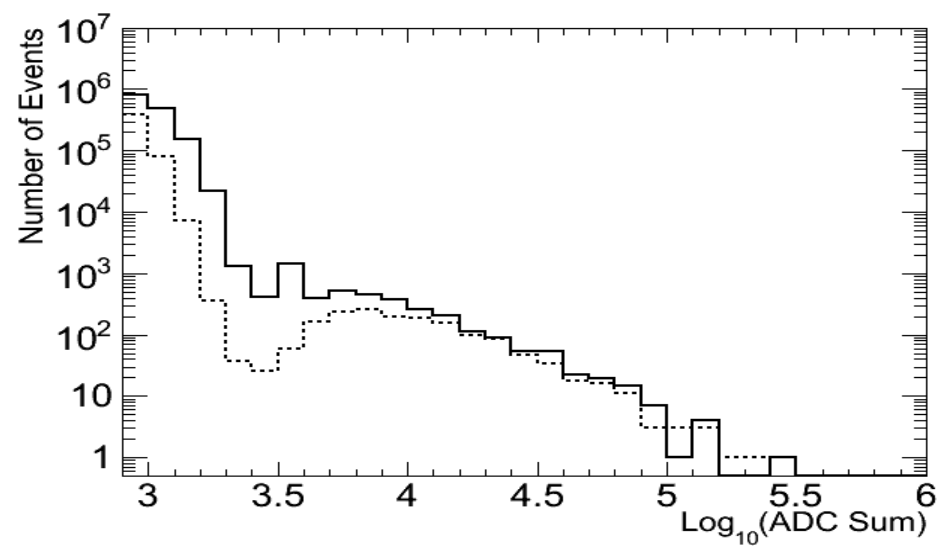

Fig. 6 ADC sum with trigger thresholds of -75 DAC (dotted line) and -35 DAC (solid line). 


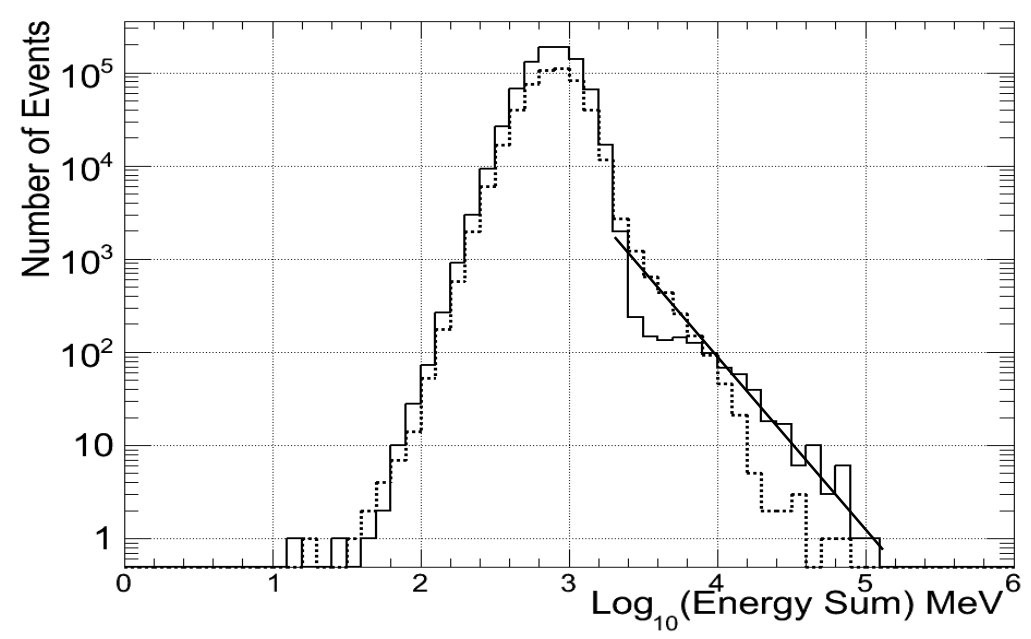

Fig. 7. Preliminary distribution of the CAL energy deposit for November 2018. The solid line represents the CAL energy deposit in $\mathrm{MeV}$ for the EHi and the dashed line represents Elow triggered events.

\section{Summary}

The ISS-CREAM instrument was successfully launched in August, 2017 and has flown one and half years in space. ISS-CREAM collected high energy cosmic rays with good preformances. The CAL is the major instrument for measuring particle energy and triggering on events with showers in the CAL. To reduce noise levels the CAL trigger threshold was adjusted during the flight. Changing from -75 DAC to -35 DAC increased frequency of the lower energy events with the lower threshold. The energy deposit distribution shows reasonable power-law behavior. Gain stitching for mid- and high-range data is still to be done. The energy deconvolution will have to be applied to account for the finite energy resolution. Further analysis would include extensive Monte Carlo simulations reported in [16] to determine efficiencies and acceptance.

\section{Acknowledgments}

This work was supported in the U.S. by NASA grant NNX17AB41G, in Korea by National Research Foundation grants 2018R1A2A1A05022685 and 2018R1A6A1A06024970, and their predecessor grants. It was also supported in France by IN2P3/CNRS and CNES and in Mexico by DGAPA-UNAM project IN109617. The authors thank NASA GSFC WFF and its contractors for engineering support and project management, JSC ISS Program Office for the launch support and ISS accommodation, MSFC for the operational support, and KSC and SpaceX for the launch support.

\section{References}

[1] E.S. Seo et al., Cosmic Ray Energetics And Mass for the International Space Station (ISS-CREAM), Adv. Sp. Res. 53, 10, 1451-1455 (2014). 
[2] M. H. Lee et al., Performance of CREAM 3 Calorimeter, IEEE Transactions on Nuclear Science, 56, 1396 - 1399 (2009).

[3] J. H. Han et al., Performance of the CREAM-V and CREAM-VI Calorimeter in flight, Proc. 32th Int. Cosmic Ray Conf. Baijing, 6, 388 (2011).

[4] I.H. Park et al., Silicon charge detector for the CREAM experiment, Nucl. Inst. Meth. A 570, 286291 (2007).

[5] J. Lee et al., Design, Fabrication and Performance of the Silicon Charge Detector for the ISSCREAM, Proc. 34th Int. Cosmic Ray Conf., The Hague, Netherlands, ID 693 (2015).

[6] Y.S. Hwang et al., Construction and Testing of a Top Counting Detector and a Bottom Counting Detector for the Cosmic Ray Energetics And Mass Experiment on the International Space Station, J. INST 10 (07), P07018 (2015).

[7] H.J. Hyun et al., Performances of photodiode detectors for top and bottom counting detectors of ISS-CREAM experiment, Nucl. Inst. Meth. A 787, 134-139 (2015).

[8] J.T. Link et al., Design and Performance of the ISS-CREAM Boronated Scintillator Detector, Proc. 34th Int. Cosmic Ray Conf., The Hague, Netherlands, ID 611 (2015).

[9] E.S. Seo et al., Cosmic-ray energetics and mass (CREAM) balloon project, Adv. In Sp. Res. 33.10, 1777-1785 (2004).

[10] H.S. Ahn et al., The Cosmic Ray Enegetics And Mass (CREAM) Instrument, Nucl. Instrum. Methods A, 579, 1034-1053 (2007).

[11] R. Takeishi et al. Cosmic-Ray Elemental Spectra Measured With ISS-CREAM, submitted to PoS(ICRC2019).

[12] J. P. Lundquist et al., Track Reconstruction for ISS-CREAM Resulting in Improved Energy and Charge Resolution, submitted to PoS(ICRC2019).

[13] H. S. Ahn et al., Performance of CREAM Calorimeter: Results of Beam Tests, Nuclear Physics B(Poc. Suppl.), 150, 272-275, (2006).

[14] J.H. Han et al., Calibration of the CREAM calorimeter with beam test data, Proc. 32nd Int. Cosmic Ray Conf., Beijing, 6, 392, (2011).

[15] N. Picot-Clemente et al., Performance of the ISS-CREAM Calorimeter, Proc. $35^{\text {th }}$ Int. Cosmic Ray Conf., Busan, Korea, 247 (2017)

[16] J. Wu et al., Monte Carlo Simulation of the ISS-CREAM Instrument, submitted to PoS(ICRC2019) 